\title{
Answers to Picture Quiz
}

A. LA $\leftrightarrow$ RA lead reversal

Look for $p$ wave inversion in $L_{1}$ and $a V L$

QRS complexes and $T$ waves are also negative in $L_{1}$ and $a V L$

$\mathrm{P}$ wave, QRS complex and T wave are positive in aVR

B. LA $\leftrightarrow$ LL lead reversal

$P$ wave and QRS complex are inverted in $L_{3}$.

$P$ wave is larger in $L 1$ than in $L_{2}$.

C. RA $\leftrightarrow R L$ lead reversal

$\mathrm{L}_{2}$ has extremely low amplitude

D. Complete reversal of chest leads $\left(V_{1} \leftrightarrow V_{6}, V_{2} \leftrightarrow V_{5}\right.$ and $\left.V_{3} \leftrightarrow V_{4}\right)$

Complete reversal of the progression of the $R$ wave.

$V_{1}$ is seen at $V 6$ and $V 6$ is seen at $V_{1}$.

$\mathrm{V}_{2}$ is seen at $\mathrm{V} 5$ and $\mathrm{V} 5$ is seen at $\mathrm{V}_{2}$.

$V_{3}$ is seen at $V 4$ and $V 4$ is seen at $V_{3}$.

\section{Normal ECG}

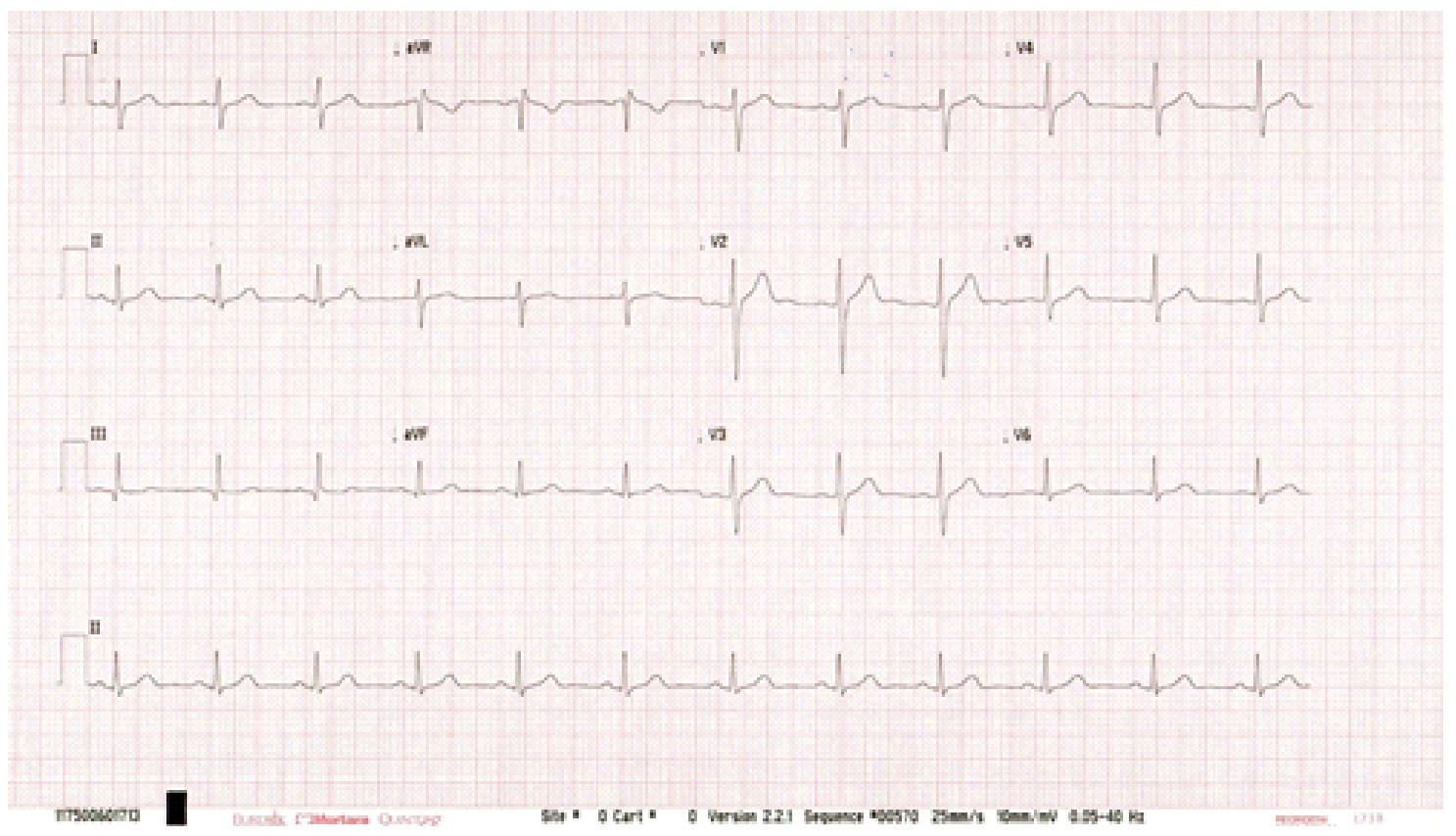
and source are credited. 